\title{
Revisão bibliométrica e apresentação de caso clínico de um fibroelastoma papilifero
}

\author{
Bibliometric review and presentation of clinical case of a fibroelastoma papilifero \\ Revisión bibliométrica y presentación de un caso clínico de fibroelastoma papilifero
}

Recebido: 13/02/2021 | Revisado: 20/02/2021 | Aceito: 22/02/2021 | Publicado: 28/02/2021

\author{
Kerlany Oliveira Carvalho \\ ORCID: https://orcid.org/0000-0001-9957-7309 \\ Instituto de Educação Superior do Vale do Parnaíba, Brasil \\ E-mail: kerlanyoc@gmail.com \\ André Luca Araujo de Sousa \\ ORCID: https://orcid.org/0000-0003-4876-9188 \\ Universidade Estadual do Piauí, Brasil \\ E-mail: a.lucaaraujo10@gmail.com \\ Ana Rachel Oliveira de Andrade \\ ORCID: https://orcid.org/0000-0002-8981-0856 \\ Instituto de Educação Superior do Vale do Parnaíba, Brasil \\ E-mail: ana.andrade@iesvap.edu.br \\ Tereza Cristina de Carvalho Souza Garcês \\ ORCID: https://orcid.org/0000-0001-6337-5166 \\ Instituto de Educação Superior do Vale do Parnaíba, Brasil \\ E-mail: tereza.garces@iesvap.edu.br \\ Leonam Costa Oliveira \\ ORCID: https://orcid.org/0000-0003-4559-7202 \\ Instituto de Educação Superior do Vale do Parnaíba, Brasil \\ E-mail: leonam.oliveira@iesvap.edu.br \\ José Lopes Pereira Júnior \\ ORCID: https://orcid.org/0000-0001-5621-7469 \\ Instituto de Educação Superior do Vale do Parnaíba, Brasil \\ E-mail: jose.junior@iesvap.edu.br \\ Nelson Cordeiro Pinheiro Sampaio \\ ORCID: https://orcid.org/0000-0002-4500-2654 \\ Instituto de Educação Superior do Vale do Parnaíba, Brasil \\ E-mail: nelson.sampaio@iesvap.edu.br \\ Antonione Santos Bezerra Pinto \\ ORCID: https://orcid.org/0000-0002-6577-2816 \\ Instituto de Educação Superior do Vale do Parnaíba, Brasil \\ E-mail: antonione.pinto@iesvap.edu.br
}

\begin{abstract}
Resumo
O presente estudo teve como proposta o aprofundamento em relação ao tumor cardíaco Fibroelastoma Papilar (FEP). O manuscrito objetivou um levantamento bibliográfico a respeito do FEP, além de realizar uma breve revisão literária sobre essa alteração patológica e apresentar um caso clinico representativo a respeito do tema. Inicialmente, o levantamento bibliográfico foi realizado na base de dados ISI Web of Knowledge/Web of Science ${ }^{\mathrm{TM}}$ sobre essa cardiopatia e seu diagnóstico, em seguida um estudo literário a respeito do tema a partir dos artigos foi apresentado e, por fim, um caso clínico de um FEP foi relatado. Dessa maneira, 214 publicações foram identificadas, onde parte delas concluíram que o FEP é uma neoplasia tumoral cardíaca que pode ser encontrada em qualquer parte do coração, sendo mais comumente localizado no endocárdio valvar e a válvula aórtica. Além da excisão cirúrgica ser o tratamento de escolha mais predominante. E conforme proposto, relatou-se um caso de uma paciente de 56 anos que apresentou o tumor na válvula aórtica e que realizou a ressecção cirúrgica, apresentando a cura. A partir dos dados apresentados, um diagnóstico precoce juntamente a um tratamento eficaz melhora o prognóstico de um paciente que apresentar essa neoplasia.
\end{abstract}

Palavras-chave: Bibliografia; Diagnóstico; Cardiopatias; Neoplasias.

\section{Abstract}

The present study proposed to deepen in relation to the Papillary Fibroelastoma Tumor (FEP). The manuscript aimed at a bibliographic survey about the FEP, in addition to performing a brief literary review on this pathological alteration and presenting a representative clinical case on the subject. Initially, the bibliographic survey was carried out on the ISI Web of Knowledge / Web of Science ${ }^{\mathrm{TM}}$ on this heart disease and its diagnosis, then a literary study on the topic from the articles was presented and, finally, a clinical case of an FEP has been reported. Thus, 214 publications were 
identified, where part of them concluded that FEP is a cardiac tumor neoplasia that can be found in any part of the heart, being more commonly located in the valve endocardium and the aortic valve. In addition, surgical excision is the most prevalent treatment of choice. And as proposed, a case of a 56-year-old patient who presented the tumor in the aortic valve and who underwent surgical resection, presenting a cure, was reported. From the data presented, an early diagnosis together with an effective treatment improves the prognosis of a patient who has this neoplasm.

Keywords: Bibliography; Diagnosis; Heart Diseases; Neoplasms.

\section{Resumen}

El presente estudio propuso profundizar en relación al Tumor de Fibroelastoma Papilar (FEP). El manuscrito tuvo como objetivo realizar un relevamiento bibliográfico sobre la PEP, además de realizar una breve revisión literaria sobre esta alteración patológica y presentar un caso clínico representativo sobre el tema. Inicialmente se realizó el relevamiento bibliográfico en la base de datos ISI Web of Knowledge / Web of ScienceTM sobre esta cardiopatía y su diagnóstico, luego se presentó un estudio literario sobre el tema a partir de los artículos y, finalmente, se ha presentado un caso clínico de una EFF. ha sido informado. Así, se identificaron 214 publicaciones, en las que parte de ellas concluyó que la FEP es una neoplasia tumoral cardíaca que se puede encontrar en cualquier parte del corazón, localizándose más comúnmente en el endocardio valvular y la válvula aórtica. Además, la escisión quirúrgica es el tratamiento de elección más prevalente. Y como se propuso, se reportó el caso de un paciente de 56 años que presentó el tumor en la válvula aórtica y que fue sometido a resección quirúrgica, presentando cura. A partir de los datos presentados, un diagnóstico precoz junto con un tratamiento eficaz mejora el pronóstico de un paciente que padece esta neoplasia.

Palabras clave: Bibliografía; Diagnóstico; Cardiopatías; Neoplasias.

\section{Introdução}

O Fibroelastoma Papilífero (FEP) é um tumor cardíaco raro inicialmente descrito como achados incidentais em autópsias ou cirurgias (Araoz et al., 2000; Bicer et al., 2009; Butany et al., 2005; Chung et al., 2020; Grinda et al., 1999; Howard et al., 1999; Mkalaluh et al., 2017). Mas também, relatórios anteriores apontaram uma associação deste tumor com a embolização cerebral, coronariana e morte súbita, assim sendo evidenciado como uma condição perigosa (Baikoussis et al., 2016; Bicer et al., 2009; Fabricius et al., 2002; Sousa-Uva \& Cardim, 2018; Taha \& Kumaresan, 2019).

No entanto, clinicamente tal alteração é mais comumente presente na forma de embolização sistêmica resultante de trombos anexados, bem como da fragmentação das próprias válvulas papilares (Araoz et al., 2000; Bruce, 2011; Topol, Biern \& Reitz, 1986). E essa neoplasia apresenta uma evolução inespecífica, com um crescimento lento e assintomático, além de apresentar um bom prognóstico nos dias atuais e passíveis de serem detectados em pacientes vivos (Baikoussis et al., 2016; Bicer et al., 2009; Butany et al., 2005; Rodrigues et al., 2018).

Histologicamente, a superfície tumoral do FEP é coberta por uma camada de células endoteliais hiperplásicas, com uma regular aderência por trombos de fibrina (Grinda et al., 1999; Sparrow et al., 2005). Além disso, seu núcleo tem a matriz constituída por colágeno, mucopolissacarídeos, fibras elásticas e raras células fusiformes com similaridade às células musculares lisas e fibroblásticas, assim tendo uma semelhança, de forma grosseira, à uma "anêmona do mar". A mobilidade desse tumor através dos pedúnculos, juntamente com os trombos aderentes e material de fibrina, representam uma fonte de êmbolo (Baikoussis et al., 2016; Casavecchia et al., 2018; Chung et al., 2020; Kumar et al., 2013; Rodrigues et al., 2018; Sousa-Uva \& Cardim, 2018; Taha \& Kumaresan, 2019).

Esse tumor pode ser encontrado em qualquer região do coração e representa cerca de $10 \%$ dos tumores nesse órgão, com predominâncias mais comuns na válvula aórtica (29\%) e válvula mitral (25\%) do que nos vasos sanguíneos pulmonares (13\%) e válvula tricúspide (17\%). Porém, cerca de 16\% das ocorrências dessa alteração surgem em superfícies não valvares (Abu et al., 2016; Al-Azizi et al., 2019; Araoz et al., 2000; Baikoussis et al., 2016; Bicer et al., 2009; Bruce, 2011; Casevecchia et al., 2018; Chung et al., 2020; Grinda et al., 1999; Mkalaluh et al., 2017; Rodrigues et al., 2018). Como características macroscópicas, o FEP apresenta uma coloração branca-amarronzada, com aspecto macio e friável. E além de possuir tamanho médio de aproximadamente $1 \mathrm{~cm}$ de diâmetro, mas podendo variar entre 0,2 a 4,6 cm, anexados ao endocárdio por uma haste 
curta e fina. Microscopicamente, essa patologia consiste em um tecido conjuntivo avascular revestido por um endotélio (Grinda et al., 1999; Rodrigues et al., 2018; Sparrow et al., 2005; Taha \& Kumaresan, 2019)

O diagnóstico é feito principalmente pelo ecodopplercardiograma transesofágico (ETE) devido ao seu alto poder de especificidade e sensibilidade, além de atualmente ser o exame de imagem mais indicado para essa etapa e caracterização do FEP (Araoz et al., 2000; Bruce, 2011; Butany et al., 2005; Chung et al., 2020; Mkalaluh et al., 2017; Reeder et al., 1991; Rodrigues et al., 2018; Taha \& Kumaresan, 2019). Após o diagnóstico, o uso de anticoagulante sistêmico é recomendado para prevenção da formação de trombos ao redor desse tumor e em casos de não sintomatologia (Baikoussis et al., 2016; Grinda et al., 1999; Mkalaluh et al., 2017)

Logo, o tratamento indicado para a neoplasia é a excisão cirúrgica, pois se faz necessário para prevenir embolias recorrentes e, assim, os índices de mortalidade serão diminuídos, além de ser relatado como seguro e curativo (Abu et al., 2016; Bruce, 2011; Butany et al., 2005; Chung et al., 2020; Grinda et al., 1999; Howard et al., 1999; Makalaluh et al., 2017; Rodrigues et al., 2018; Sousa-Uva \& Cardim, 2018; Taha \& Kumaresan, 2019). E dependendo do grau de comprometimento das válvulas cardíacas, em muitos dos casos é preciso fazer uma reposição estrutural e troca da mesma a cada 10 anos ou menos, e as vezes não é necessário fazer a troca da válvula (howard et al., 1999). Contudo, Bobadilha et al. (2017) afirma que essa alteração cardíaca pode ser excisada com preservação valvar e com bom prognóstico a médio prazo.

O presente estudo teve como objetivos elaborar um levantamento bibliográfico a respeito do Diagnóstico do Tumor Fibroelastoma Palilífero (FEP) e, a partir da análise de alguns desses artigos, realizar uma breve revisão de literatura a respeito dessa patologia, além de consolidar o assunto com um relato de caso clínico elucidando o diagnóstico dessa alteração através de exames de imagens, além do acompanhamento pós-operatório do paciente.

\section{Metodologia}

Este estudo trata-se de uma pesquisa quantitativa e qualitativa a respeito do tumor benigno Fibroelastoma Papilífero (Koche, 2011; Pereira et al., 2018). Dessa forma, realizou-se um mapeamento sobre as produções científicas internacionais sobre o FEP. E foram usados os termos de busca: "papillary fibroelastoma*" and "diagnosis" - o asterisco possibilita a busca nos plurais das palavras - em uma das principais bases de dados, a ISI Web of Knowledge/Web of Science ${ }^{\mathrm{TM}}$, no recorte temporal entre os anos 1945 e 2018 e analisando os registros que apresentam os termos selecionados para a busca, com base em revisão de literatura sobre a temática em questão. Como percurso metodológico, foi realizada uma pesquisa bibliométrica em trabalhos acadêmicos na Web of Science ${ }^{\mathrm{TM}}$. Assim, como principais resultados das análises foram identificados a quantidade de artigos em evolução, os “top journals” da temática, e os artigos mais citados.

Após realizado o levantamento bibliométrico na principal coleção do Web of Science ${ }^{\mathrm{TM}}$ foram identificados 214 artigos sobre o FEP. Estes artigos estão publicados em 125 periódicos distintos indexados à base de dados em questão e foram escritos por 1080 autores que possuem vínculos à 282 instituições, localizadas em 42 países. Para a consecução destes artigos foram utilizadas 1.998 referências, com uma média de aproximadamente 9,3 referências por artigo.

Após análise dos artigos e descrição dos resultados tabelados, uma apresentação de caso clínico de um paciente com Fibroeslastoma Papilífero foi relatada com a finalidade de elucidar o diagnóstico e o pós-tratamento dessa neoplasia. Assim, todos os parâmetros éticos foram seguidos através da assinatura de um Termo de Consentimento Livre e Esclarecido (TCLE) de forma prévia.

\section{Levantamento Bibliográfico}

- Busca para anos completos: 1945-2018 (mas o primeiro registro de artigo resultado da busca foi em 1986); 
- Citações indexadas: Todos os indexes da principal coleção da Web of Science ${ }^{\mathrm{TM}}$ à exceção dos conference proceedings;

- $\quad$ Tipos de documentos: apenas artigos e revisões; todos os materiais editoriais, capítulos de livros e artigos em eventos foram excluídos;

- $\quad$ Resultados: 214 produções científicas.

Na Tabela 1, a seguir, são apresentados esses resultados.

Tabela 1: Resultados Gerais do Levantamento Bibliométrico (1986-2018).

\begin{tabular}{lc}
\hline \multicolumn{1}{c}{ Dados Bibliométricos } & Quantidade \\
\hline Publicações (artigos) & 214 \\
\hline Periódicos indexados & 125 \\
\hline Autores & 1080 \\
Instituições (vínculos dos autores) & 282 \\
\hline Países & 42 \\
\hline Referências citadas & 1998 \\
\hline
\end{tabular}

Fonte: Elaboração própria a partir de dados da Web of Science ${ }^{T M}$.

Figura 1: Distribuição das publicações (1986-2018).

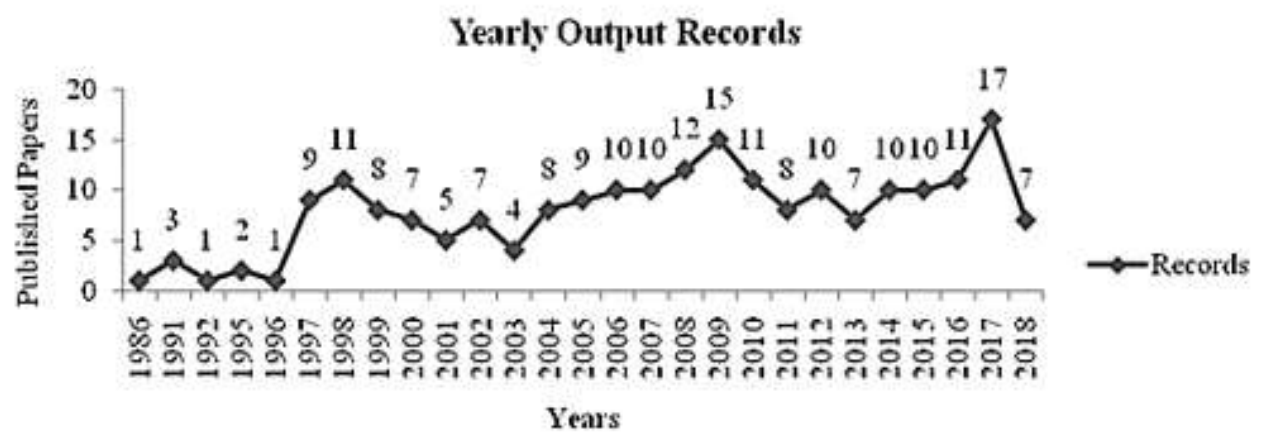

Distribuição Anual de Frequência de Publicações

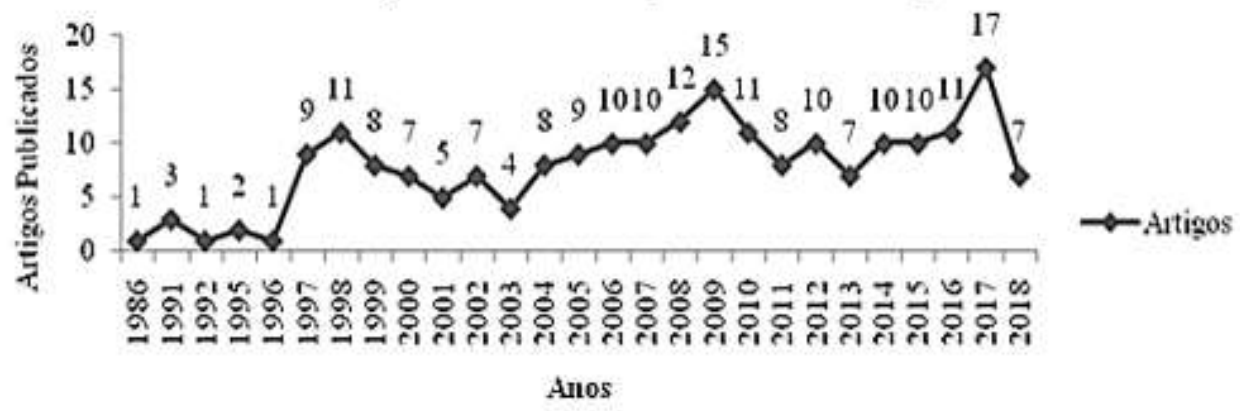

Distribuição dos artigos em inglês e português. Fonte: Elaboração própria a partir de dados da Web of Science. 
Research, Society and Development, v. 10, n. 2, e57210212942, 2021 (CC BY 4.0) | ISSN 2525-3409 | DOI: http://dx.doi.org/10.33448/rsd-v10i2.12942

Tabela 2: Distribuição anual das publicações (1986-2018).

\begin{tabular}{|c|c|c|c|c|c|}
\hline Ano & Artigos & Citações & Ano & Artigos & Citações \\
\hline 1986 & 1 & 83 & 2006 & 10 & 58 \\
\hline 1991 & 3 & 107 & 2007 & 10 & 72 \\
\hline 1992 & 1 & 14 & 2008 & 12 & 64 \\
\hline 1995 & 2 & 51 & 2009 & 15 & 225 \\
\hline 1996 & 1 & 17 & 2010 & 11 & 97 \\
\hline 1997 & 9 & 337 & 2011 & 8 & 183 \\
\hline 1998 & 11 & 101 & 2012 & 10 & 78 \\
\hline 1999 & 8 & 252 & 2013 & 7 & 14 \\
\hline 2000 & 7 & 345 & 2014 & 10 & 28 \\
\hline 2001 & 5 & 283 & 2015 & 10 & 20 \\
\hline 2002 & 7 & 51 & 2016 & 11 & 38 \\
\hline 2003 & 4 & 58 & 2017 & 17 & 19 \\
\hline 2004 & 8 & 25 & 2018 & 7 & 8 \\
\hline 2005 & 9 & 539 & - & - & - \\
\hline
\end{tabular}

Fonte: Elaboração própria a partir de dados da Web of Science ${ }^{\mathrm{TM}}$. 
Tabela 3: Top Periódicos com mais artigos publicados (1986-2018).

\begin{tabular}{|c|c|c|}
\hline Periódicos & $\begin{array}{c}\text { Quantidade de } \\
\text { Artigos }\end{array}$ & Citações \\
\hline Texas Heart Institute Journal & 15 & 134 \\
\hline $\begin{array}{l}\text { Echocardiography - A Journal of Cardiovascular Ultrasound } \\
\text { and Allied Techniques }\end{array}$ & 10 & 71 \\
\hline Journal of Cardiothoracic Surgery & 7 & 52 \\
\hline Journal of Heart Valve Diseade & 7 & 90 \\
\hline Cardiovascular Pathology & 6 & 64 \\
\hline Annals of Thoracic Surgery & 5 & 147 \\
\hline Archives des Maladies du Coeur et des Vaisseaux & 5 & 22 \\
\hline Canadian Journal of Cardiology & 5 & 38 \\
\hline Heart Surgery Forum & 4 & 10 \\
\hline Journal of the American Society of Echcardiography & 4 & 24 \\
\hline
\end{tabular}

Fonte: Elaboração própria a partir de dados da Web of Science.

Tabela 4: Autores com maior número de publicações (1986-2018)

\begin{tabular}{ccccc}
\hline Autores & Artigos & Citações & Afiliação (Instituição de vínculo) & País \\
\hline Berrebi A & 3 & 117 & Pierre and Marie Curie University & França \\
Chauvaud S & 3 & 117 & Pierre and Marie Curie University & Alemanha \\
Diegeler A & 3 & 18 & Campus Bad Neustadt der RHÖN-KLINIKUM & Canadá \\
\hline Veinot JP & 3 & 30 & University of Ottawa &
\end{tabular}

Fonte: Elaboração própria a partir de dados da Web of Science $e^{T M}$.

A tabela com os autores foi elaborada a partir da classificação deles pelo total de publicações e total de citações recebidas. Os demais autores, da $5^{\mathrm{a}}$ à $64^{\mathrm{a}}$ posição apresentaram dois artigos como resultado. Os demais listados a partir da $65^{\mathrm{a}}$ posição apresentaram 1 artigo 
Research, Society and Development, v. 10, n. 2, e57210212942, 2021

(CC BY 4.0) | ISSN 2525-3409 | DOI: http://dx.doi.org/10.33448/rsd-v10i2.12942

Tabela 5: Quantidade de artigos por país de origem das instituições de vínculo dos autores.

\begin{tabular}{lccc}
\hline País & Quantidade & Citações \\
\hline USA & 67 & 1690 & 137 \\
Alemanha & 19 & 192 \\
Itália & 18 & 239 \\
França & 16 & 51 \\
Japão & 11 & 439 \\
Canadá & 10 & 20 \\
China & 7 & 26 \\
Espanha & 7 & 66 \\
Bélgica & 6 & 136 \\
Suíça & 6 & 66
\end{tabular}

Fonte: Elaboração própria a partir de dados da Web of Science ${ }^{T M}$. 
Tabela 6: Artigos mais citados.

\begin{tabular}{|c|c|c|c|}
\hline Autores/ano & Título & Fonte & Citações \\
\hline Butany et al. (2005) & Cardiac tumours: diagnosis and management & Lancet Oncology & 319 \\
\hline Sun et al. (2001) & $\begin{array}{l}\text { Clinical and echocardiographic characteristics of } \\
\text { papillary fibroelastomas }-a \text { retrospective and } \\
\text { prospective study in } 162 \text { patients }\end{array}$ & Circulation & 236 \\
\hline Klarich et al. (1997) & $\begin{array}{l}\text { Papillary fibroelastoma: echocardiographic } \\
\text { characteristics for diagnosis and pathologic correlation }\end{array}$ & $\begin{array}{l}\text { Journal of the American } \\
\text { College of Cardiology }\end{array}$ & 204 \\
\hline Araoz et al. (2000) & $\begin{array}{l}\text { CT and MR imaging of benign primary cardiac } \\
\text { neoplasms with echo cardiographic correlation }\end{array}$ & Radiographics & 188 \\
\hline Sparrow et al. (2005) & MR imaging of cardiac tumors & Radiographics & 180 \\
\hline Grinda et al. (1999) & $\begin{array}{l}\text { Cardiac valve papillary fibroelastoma: surgical } \\
\text { excision for revealed or potential embolization }\end{array}$ & $\begin{array}{l}\text { Journal of Thoracic and } \\
\text { Cardiovascular Surgery }\end{array}$ & 102 \\
\hline Bruce (2011) & Cardiac tumours: diagnosis and management & Heart & 92 \\
\hline Topol et al. (1991) & $\begin{array}{l}\text { Cardiac papillary fibroelastoma and stroke - } \\
\text { echocardiographic diagnosis and guide to excision }\end{array}$ & $\begin{array}{l}\text { American Journal of } \\
\text { Medicine }\end{array}$ & 83 \\
\hline Reeder et al. (1991) & $\begin{array}{l}\text { Transesophageal echocardiographic and cardiac } \\
\text { masses }\end{array}$ & Mayo Clinic Proceedings & 83 \\
\hline Howard et al. (1999) & $\begin{array}{l}\text { Papillary fibroelastoma: increasing recognition of a } \\
\text { surgical disease }\end{array}$ & Annals of Thoracic Surgery & 83 \\
\hline
\end{tabular}

Fonte: Elaboração própria a partir de dados da Web of Science ${ }^{T M}$.

\section{Caso Clínico}

Paciente do gênero feminino, de 55 anos, compareceu a clínica médica com queixas álgicas torácicas constantes. Além da paciente não ser usuária de drogas lícitas e/ou ilícitas. 
$\mathrm{Na}$ região do tórax, uma tomografia computadorizada (TC) foi realizada e, em seguida, obteve-se um sugestivo diagnóstico de bronquite crônica após análise (Figura 1). Contudo, depois de uma nova avaliação com um cardiologista, houve uma sinalização de cisto na válvula aórtica.

Figura 1: Tomografia Computadorizada em cortes axiais.
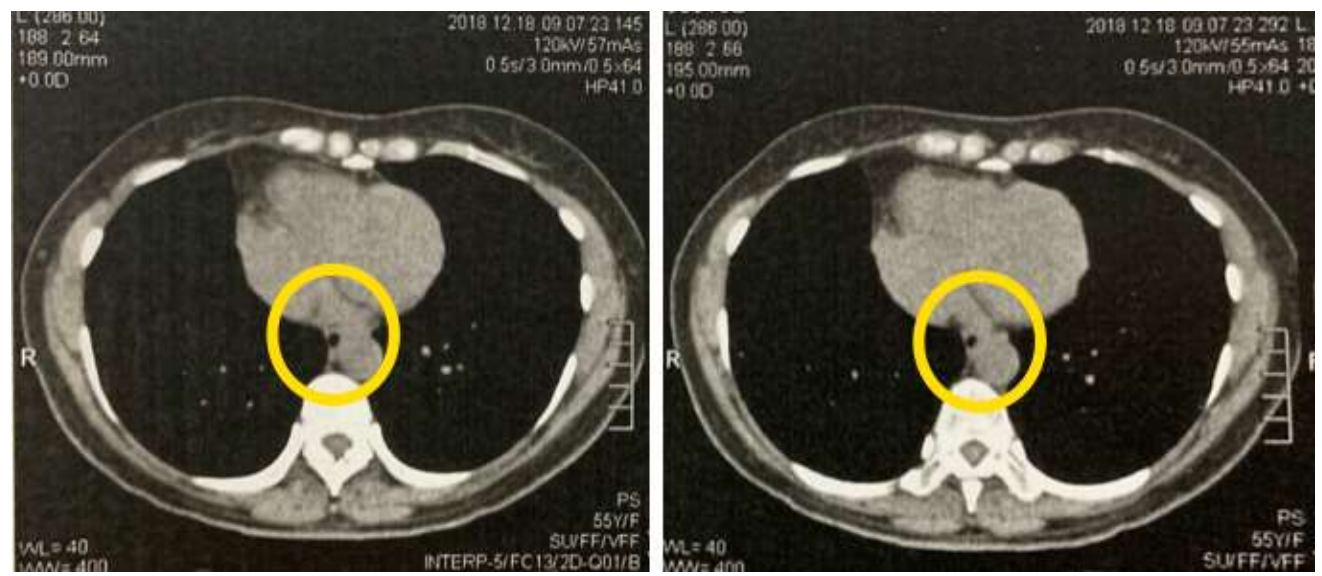

Os cortes revelam pequena placa calcificada no arco da aorta. Fonte: Arquivo pessoal.

Logo, uma angiotomografia computadorizada (AC) e Ecodopplercardiograma Transesofagico (ETE) foram executadas. Dessa maneira, tais exames espelharam uma imagem pedunculada, móvel, com dimensões aproximadas de 8,2x7,1 mm, aderida à área do folheto aórtico não coronariano e, assim, não determinando disfunção valvar (Figura 2). Logo, as imagens propiciaram o diagnóstico de Fibroelastoma Papilífero (FEP) na válvula aórtica.

Como tratamento, a excisão cirúrgica do tumor foi optada, além do auxílio medicamentoso com ácido acetilsalicílico $81 \mathrm{mg}$ e encaminhamento ao especialista na área de cardiopatia. Em seguida, a paciente foi submetida à exames pré-operatórios como eletrocadiograma, radiografia de tórax póstero-anterior e lateral, além de um ecocardiograma com mapeamento de fluxo em cores (Figura 2).

Figura 2: Ecodopplercardiograma transesofágico tridimensional.

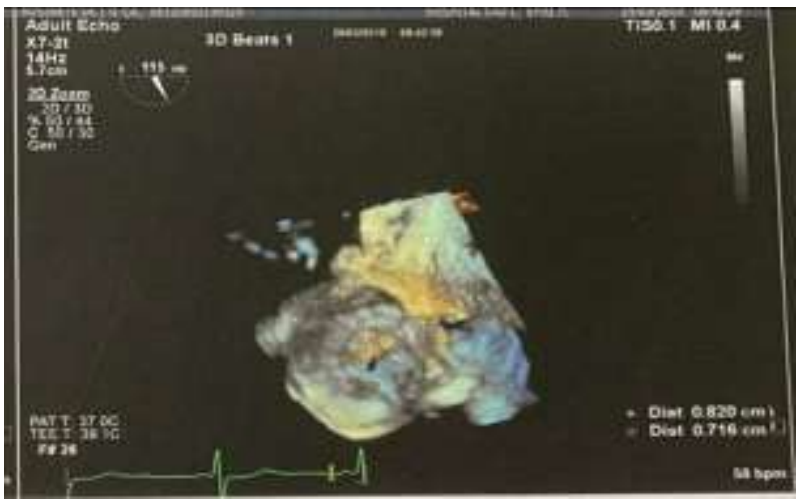

Diferentes parâmetros mostram a imagem do Fibroelastoma Papilífero.

Fonte: Arquivo pessoal. 
Após ao procedimento cirúrgico de remoção do FEP de aproximadamente $12 \mathrm{~mm}$, sem necessidade de abordagem da válvula aórtica, realizou-se estudo anatomopatológico da lesão de consistência elástica, coloração branca e com dois fragmentos irregulares (Figura 3).

Figura 3: Tumor resseccionado em bloco.

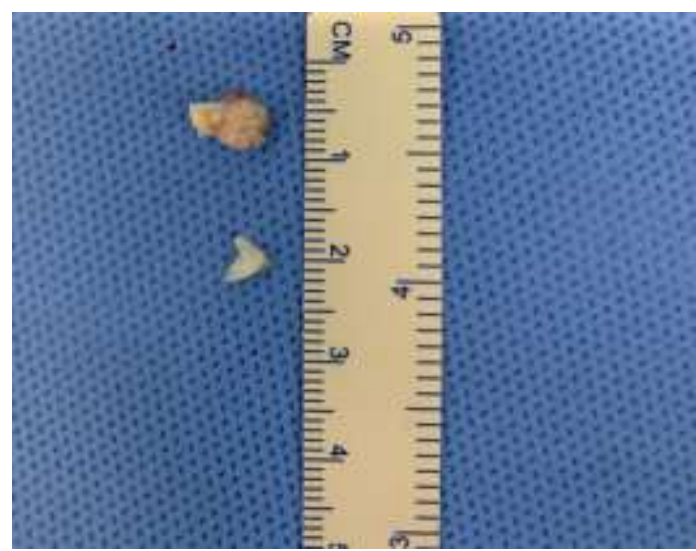

Tumor com dimensões de 1,4 x 0,5 x 0,4 cm. Fonte: Arquivo pessoal.

Em relação à avalição histopatológica, os cortes revelaram na coloração com hematoxilina-esosina formações arborescentes com eixo de tecido conjuntivo. Na parte central eosinofílica, um padrão histológico proteoglicano estava presente. Porém, pedículos curtos de tecido conjuntivo denso e coberto por células endoteliais hiperplásicas também eram notados. E por fim, células conectivas soltas do núcleo do tecido continha colágeno e fibras elásticas, com células musculares lisas ocasionais (Figura 4).

Figura 4: Cortes histológicos.
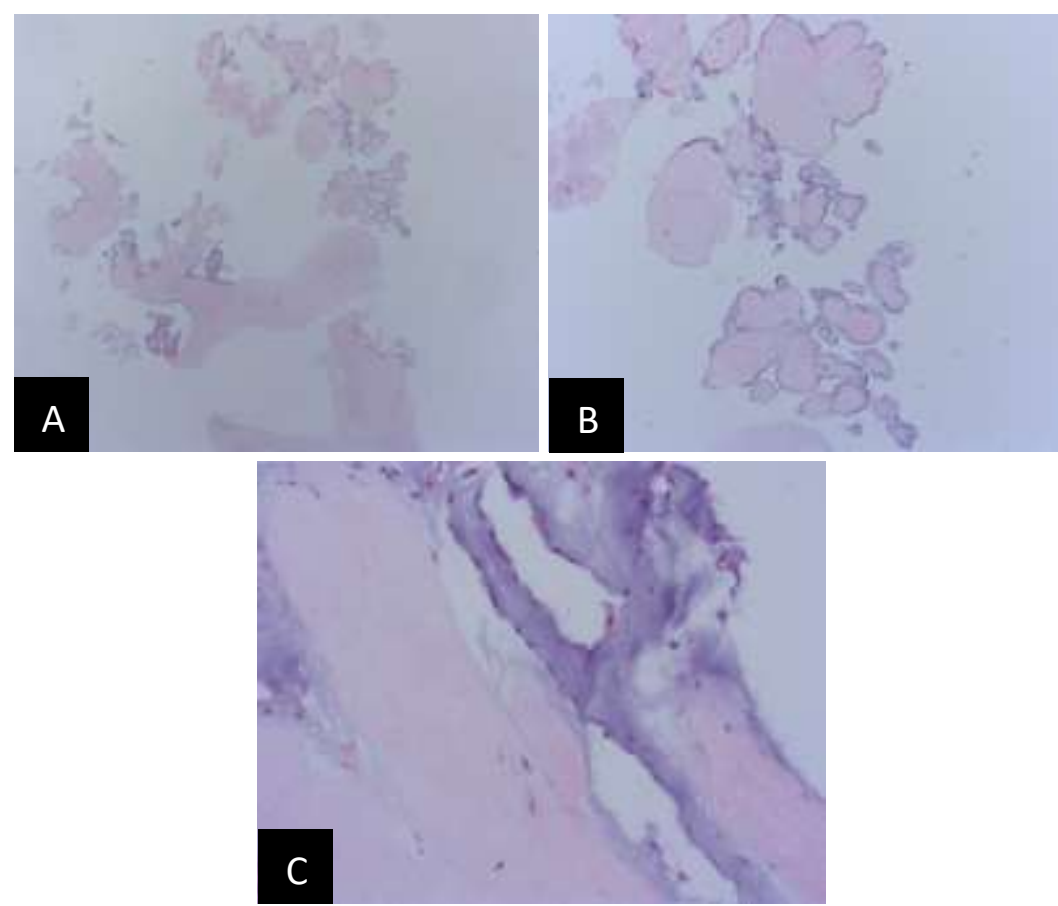

A, B e C: imagens com aumento de 4x, 4x e 40x respectivamente, cujos cortes histológicos apresentam presença de revestimento por endocárdio, com células fusiformes de características de fibroblasto 
ou miofibroblastos em posição mais central.

Fonte: Arquivo pessoal.

Durante o pós-operatório, a paciente foi submetida a terapia complementar com drenagem torácica e prescrições medicamentosas, mas também, fisioterapia respiratória diária e transfusões sanguíneas devido a um estado anêmico. Durante esse período de internação hospitalar, cefaleias e dores ao tossir eram constadas. Logo após a alta hospitalar, a mesma foi submetida a um ecodopplercardiograma transtorácico de acompanhamento que apresentou imagens dentro dos parâmetros normais (Figura 2). Dessa forma, sem intercorrências durante a recuperação e com acompanhamento clinico contínuo.

\section{Discussão}

Nos estudos realizados por Mkalaluh et al. (2017)e Abu Saleh et al. (2016) aproximadamente metade dos pacientes com o FEP apresentavam queixas de dores no peito, embora a causa fosse difícil de estabelecer. Assim, a sintomatologia foi semelhante ao do presente relato de caso.

Dentro dos estudos radiológicos realizados durante o processo de diagnóstico do FEP, as imagens obtidas pela TC não foram suficientes para visualizar essa alteração, pois a patologia apresentava dimensões pequenas e acoplagem às válvulas móveis (Araoz et al., 2000). Assim, exames de alta especificidade e acurácia foram necessários, além de exames de maior precisão. Mas estudos demonstram que esse tumor pode ser detectado utilizando um único exame de imagem de forma isolado ou combinado com outros, assim como realizado nesse caso onde realizou-se vários exames (Araoz et al., 2000; Baikoussis et al., 2016; Butany et al., 2005; Bruce, 2011). Dessa maneira, o ecodopplercardiograma transesofágico (ETE) se mostrou eficaz na visualização dessa alteração e consequentemente, um diagnóstico preciso foi realizado.

A histopatologia do FEP do presente caso evidenciou presenças de revestimento por endocárdio, com existência de células e tecido conjuntivo com os aspectos fusiformes. Esse achado foi semelhante ao estudo feito por Sousa-Uva e Cardim (2018) e Kumar et al. (2013).

A escolha pela excisão cirúrgica foi similar ao estudo clínico realizado por Grinda et al. (1999) que demonstrou a eficácia de $100 \%$ no tratamento cirúrgico com abordagem conservadora em 4 pacientes, sem a necessidade de transplante valvar. E Butany et al. (2005) demonstrou que esse método eleva o prognóstico do paciente, além de ser um procedimento indicado por essa cardiopatia apresentar um potencial de riscos de complicações à vida (Abu Saleh et al., 2016; Baikoussis et al., 2016; Bruce, 2011; Butany et al., 2005; Grinda et al., 1999; Howard et al., 1999; Rodrigues et al., 2018; Sousa-Uva \& Cardim, 2018).

E de acordo com os dados obtidos por Mkalaluh et al. (2017), o prognóstico dos pacientes são excelentes se observados a longo prazo e com baixos indícios de recorrência. Dessa maneira, justificando as não intercorrências durante a recuperação da paciente.

\section{Considerações Finais}

O FEP é um tumor benigno geralmente assintomático e diagnosticado ocasionalmente através de exames feitos com finalidade de avaliar outras alterações patológicas. Onde existem várias bases de dados que debatem e relatam o tema.

O uso do Ecodopplercardiograma Transesofágico é considerado o mais sensível para a análise precisa do FEP.

E a excisão cirurgia simples é a escolha de tratamento mais frequente entre os autores com ou sem transplante da válvula acometida, dependendo de cada caso. Dessa forma, várias complicações embólicas são prevenidas devido ao deslocamento do tumor. 
Portanto, tais dados auxiliam no diagnóstico precoce e consequentemente eleva um bom prognóstico para com o paciente. Dessa maneira, ainda são necessários mais estudos para tentar descobrir ou elaborar novas medidas auxiliares para fortalecer no diagnóstico.

\section{Referências}

Abu Saleh, W. K., Al Jabbari, O., Ramlawi, B., \& Reardon, M. J. (2016). Cardiac Papillary Fibroelastoma: Single-Institution Experience with 14 Surgical Patients. Texas Heart Institute journal, 43(2), 148-151. https://doi.org/10.14503/THIJ-14-4889

Al-Azizi, K. M., Hamandi, M., Baxter, R., Krueger, A., Crawford, A. W., William, M., Good, C., \& Mead, N. (2019). Papillary Fibroelastoma of the Ascending Aorta. Journal of investigative medicine high impact case reports, 7, 2324709619840377. https://doi.org/10.1177/2324709619840377

Araoz, P. A., Mulvagh, S. L., Tazelaar, H. D., Julsrud, P. R., \& Breen, J. F. (2000). CT and MR imaging of benign primary cardiac neoplasms with echocardiographic correlation. Radiographics: a review publication of the Radiological Society of North America, Inc,20(5), 1303-1319. https://doi.org/10.1148/radiographics.20.5.g00se121303

Baikoussis, N. G., Dedeilias, P., Argiriou, M., Argiriou, O., Vourlakou, C., Prapa, E., \& Charitos, C. (2016). Cardiac papillary fibroelastoma; when, how, why? Annals of cardiac anaesthesia, 19(1), 162-165. https://doi.org/10.4103/0971-9784.173040

Bicer, M., Cikirikcioglu, M., Pektok, E., Müller, H., Dettwiler, S., \& Kalangos, A. (2009). Papillary fibroelastoma of the left atrial wall: a case report. Journal of cardiothoracic surgery, 4, 28. https://doi.org/10.1186/1749-8090-4-28

Bobadilla, P., Vigliano, C., Casabé, J. H., Guevara, E., Salmo, F., Abud, J., Dulbecco, E., \& Favaloro, R. R. (2017). Fibroelastoma papilar cardíaco: estudio retrospectivo. Presentación clínica y resultados quirúrgicos [Papillary fibroelastoma: retrospective analysis. Clinical presentation and surgical results]. Medicina (B Aires), 77(6), 481-485. http://ref.scielo.org/gbdxgp

Bruce C. J. (2011). Cardiac tumours: diagnosis and management. Heart (British Cardiac Society), 97(2), 151-160. https://doi.org/10.1136/hrt.2009.186320

Butany, J., Nair, V., Naseemuddin, A., Nair, G. M., Catton, C., \& Yau, T. (2005). Cardiac tumours: diagnosis and management. The Lancet. Oncology, 6(4), 219-228. https://doi.org/10.1016/S1470-2045(05)70093-0

Casavecchia, G., Gravina, M., Zicchino, S., Moranti, E., Cuculo, A., Macarini, L., Di Biase, M., \& Brunetti, N. D. (2018). Tricuspid Papillary Fibroelastoma at Multimodal Imaging. Journal of cardiovascular echography, 28(4), 236-238. https://doi.org/10.4103/jcecho.jcecho_48_18

Chung, E. S., Lee, J. H., Seo, J. K., Kim, B. G., Kim, G. S., Lee, H. Y., Byun, Y. S., \& Kim, H. J. (2020). Early surgical in tervention for unusually located cardiac fibroelastomas. Yeungnam University journal of medicine, 37(4), 345-348. https://doi.org/10.12701/yujm.2020.00556

Fabricius, A. M., Heidrich, L., Gutz, U., \& Mohr, F. W. (2002). Papillary fibroelastoma of the tricuspid valve chordae with a review of the literature. Cardiovascular journal of South Africa: official journal for Southern Africa Cardiac Society [and] South African Society of Cardiac Practitioners, 13(3), 122-124.

Grinda, J. M., Couetil, J. P., Chauvaud, S., D'Attellis, N., Berrebi, A., Fabiani, J. N., Deloche, A., \& Carpentier, A. (1999). Cardiac valve papillary fibroelastoma: surgical excision for revealed or potential embolization. The Journal of thoracic and cardiovascular surgery, 117(1), 106-110. https://doi.org/10.1016/s00225223(99)70474-5

Howard, R. A., Aldea, G. S., Shapira, O. M., Kasznica, J. M., \& Davidoff, R. (1999). Papillary fibroelastoma: increasing recognition of a surgical disease. The Annals of thoracic surgery, 68(5), 1881-1885. https://doi.org/10.1016/s0003-4975(99)00860-7

Koche, J. C. (2011). Fundamentos da Metodologia Científica. Editora Vozes. http://www.brunovivas.com/wp-content/uploads/sites/10/2018/07/K\%C3\%B6cheJos\%C3\%A9-Carlos0D0AFundamentos-de-metodologia-cient\%C3\%ADfica-_teoria-da0D0Aci\%C3\%AAncia-e-inicia\%C3\%A7\%C3\%A3o-\%C3\%A0pesquisa.pdf

Kumar, P., Gandhi, I., Sharma, P. B., \& Ambasta, K. (2013). Why our heart is not prone to cancer? American Journal of Research Communication, 1(7), 143151. https://www.researchgate.net/publication/262005359_Why_our_heart_is_not_prone_to_cancer

Mkalaluh, S., Szczechowicz, M., Torabi, S., Dib, B., Sabashnikov, A., Mashhour, A., Karck, M., \& Weymann, A. (2017). Surgery for Cardiac Papillary Fibroelastoma: A 12-Year Single Institution Experience. Medical science monitor basic research, 23, 258-263. https://doi.org/10.12659/msmbr.904881

Pereira, A. S., Shitsuka, D. M., Parreira, F. J., \& Shitsuka, R. (2018). Metodologia da Pesquisa Científica. Núcleo de Tecnologia Educacional - UFSM. https://www.ufsm.br/app/uploads/sites/358/2019/02/Metodologia-da-Pesquisa-Cientifica_final.pdf

Reeder, G. S., Khandheria, B. K., Seward, J. B., \& Tajik, A. J. (1991). Transesophageal echocardiography and cardiac mas ses. Mayo Clinic proceedings, 66(11), 1101-1109. https://doi.org/10.1016/s0025-6196(12)65788-7

Rodrigues, J. D., Ferreira, J., Almeida, J., Campelo, M., Maciel, M. J., \& Pinho, P. (2018). Cardiac papillary fibroelastoma: Report of a surgical series. Revista portuguesa de cardiologia : orgao oficial da Sociedade Portuguesa de Cardiologia = Portuguese journal of cardiology : an official journal of the Portuguese Society of Cardiology, 37(12), 981-986. https://doi.org/10.1016/j.repc.2018.02.011

Sousa-Uva, M., \& Cardim, N. (2018). Cardiac papillary fibroelastoma: So small and yet so dangerous. Revista portuguesa de cardiologia : orgao oficial da Sociedade Portuguesa de Cardiologia = Portuguese journal of cardiology : an official journal of the Portuguese Society of Cardiology, 37(12), 987-989. https://doi.org/10.1016/j.repc.2018.11.006 
Research, Society and Development, v. 10, n. 2, e57210212942, 2021

(CC BY 4.0) | ISSN 2525-3409 | DOI: http://dx.doi.org/10.33448/rsd-v10i2.12942

Sparrow, P. J., Kurian, J. B., Jones, T. R., \& Sivananthan, M. U. (2005). MR imaging of cardiac tumors. Radiographics : a review publication of the Radiological Society of North America, Inc, 25(5), 1255-1276. https://doi.org/10.1148/rg.255045721

Taha, M. E., \& Kumaresan, J. (2019). Aortic Valve Papillary Fibroelastoma: A Sea Anemone in the Heart, A Case Report. Cardiology research, 10(6), 378381. https://doi.org/10.14740/cr818

Topol, E. J., Biern, R. O., \& Reitz, B. A. (1986). Cardiac papillary fibroelastoma and stroke. Echocardiographic diagnosis and guide to excision. The American journal of medicine, 80(1), 129-132. https://doi.org/10.1016/0002-9343(86)90063-x 